\title{
Recurrent fusions in PLAGL1 define a distinct subset of pediatric-type supratentorial ependymoma
}

Philipp Sievers ${ }^{1,2 \#}$, Sophie C. Henneken ${ }^{3,4 \#}$, Christina Blume ${ }^{1,2,5}$, Martin Sill $^{3,4}$, Daniel Schrimpf ${ }^{1,2}$, Damian Stichel ${ }^{1,2}$, Konstantin Okonechnikov ${ }^{3,4}$, David E. Reuss ${ }^{1,2}$, Julia Benzel ${ }^{3,4}$, Kendra Maaß ${ }^{3,4}$, Marcel Kool ${ }^{3,4,6}$, Dominik Sturm ${ }^{3,7,8}$, Patricia Kohlhof-Meinecke ${ }^{9}$, Ofelia Cruz ${ }^{10}$, Mariona Suñol ${ }^{11}$, Cinzia Lavarino ${ }^{12}$, Viktoria Ruf ${ }^{13}$, Henning B. Boldt ${ }^{14,15}$, Mélanie Pagès ${ }^{16,17}$, Celso Pouget ${ }^{18}$, Leonille Schweizer ${ }^{19,20}$, Mariëtte E. G. Kranendonk ${ }^{6,21}$, Noreen Akhtar22,23, Ulrich Schüller24,25,26, Wolf C. Mueller27, Hildegard Dohmen², Till Acker ${ }^{28}$, Patrick N. Harter 29,30,31,32, Christian Mawrin ${ }^{33}$, Rudi Beschorner ${ }^{34}$, Sebastian Brandner ${ }^{35,36}$, Matija Snuderl ${ }^{37}$, Zied Abdullaev ${ }^{38}$, Kenneth Aldape $^{38}$, Mark R. Gilbert ${ }^{39}$, Terri S. Armstrong ${ }^{39}$, David W. Ellison ${ }^{40}$, David Capper ${ }^{19,20}$, Koichi Ichimura ${ }^{41}$, Guido Reifenberger ${ }^{42,43}$, Richard G. Grundy ${ }^{44}$, Nada Jabado $45,46,47$, Lenka Krskova ${ }^{48,49}$, Michal Zapotocky ${ }^{48,50}$, Ales Vicha ${ }^{48,50}$, Pascale Varlet ${ }^{16}$, Pieter Wesseling ${ }^{6,51}$, Stefan Rutkowski ${ }^{25}$, Andrey Korshunov ${ }^{1,2,3}$, Wolfgang Wick ${ }^{52,53}$, Stefan M. Pfister ${ }^{3,4,8}$, David T. W. Jones ${ }^{3,7}$, Andreas von Deimling ${ }^{1,2}$, Kristian W. Pajtler ${ }^{3,4,8 \#, *}$, Felix Sahm ${ }^{1,2,3 \#, *}$

${ }^{1}$ Department of Neuropathology, Institute of Pathology, University Hospital Heidelberg, Heidelberg, Germany

${ }^{2}$ Clinical Cooperation Unit Neuropathology, German Consortium for Translational Cancer Research (DKTK), German Cancer Research Center (DKFZ), Heidelberg, Germany

${ }^{3}$ Hopp Children's Cancer Center Heidelberg (KiTZ), Heidelberg, Germany

${ }^{4}$ Division of Pediatric Neurooncology, German Cancer Consortium (DKTK), German Cancer Research Center (DKFZ), Heidelberg, Germany

${ }^{5}$ Bioinformatics and Omics Data Analytics, German Cancer Research Center (DKFZ), Heidelberg, Germany

${ }^{6}$ Princess Máxima Center for Pediatric Oncology, Utrecht, The Netherlands

${ }^{7}$ Pediatric Glioma Research Group, German Cancer Research Center (DKFZ), Heidelberg, Germany

${ }^{8}$ Department of Pediatric Oncology, Hematology, Immunology and Pulmonology, University Hospital Heidelberg, Heidelberg, Germany

${ }^{9}$ Department of Pathology, Klinikum Stuttgart, Stuttgart, Germany

${ }^{10}$ Department of Pediatric Oncology, Hospital Sant Joan de Déu, Esplugues de Llobregat, Barcelona, Spain

${ }^{11}$ Department of Pathology, Hospital Sant Joan de Déu, Esplugues de Llobregat, Barcelona, Spain

${ }^{12}$ Laboratory of Molecular Oncology, Hospital Sant Joan de Déu, Esplugues de Llobregat, Barcelona, Spain

${ }^{13}$ Institute of Neuropathology, Ludwig-Maximilian University, Munich, Germany

${ }^{14}$ Department of Pathology, Odense University Hospital, Odense, Denmark

${ }^{15}$ Department of Clinical Research, University of Southern Denmark, Odense, Denmark

${ }^{16}$ Department of Neuropathology, GHU Paris Psychiatry and Neurosciences, Sainte-Anne Hospital, Paris, France

${ }^{17}$ Laboratory of Translational Research in Pediatric Oncology, SIREDO, INSERM U830, Institut Curie, Paris Sciences Lettres University, Paris, France

${ }^{18}$ Department of Pathology, CHRU, Nancy, France

${ }^{19}$ Charité - Universitätsmedizin Berlin, corporate member of Freie Universität Berlin and Humboldt-Universität zu Berlin, Institute of Neuropathology, Berlin, Germany

${ }^{20}$ German Cancer Consortium (DKTK), Partner Site Berlin, German Cancer Research Center (DKFZ), Heidelberg, Germany

${ }^{21}$ Department of Pathology, University Medical Center Utrecht, Utrecht, The Netherlands

${ }^{22}$ Manchester Royal Infirmary, Manchester University NHS Foundation Trust, Manchester, UK

${ }^{23}$ Shaukat Khanum Memorial Cancer Hospital and Research Centre, Lahore, Pakistan

${ }^{24}$ Institute of Neuropathology, University Medical Center Hamburg-Eppendorf, Hamburg, Germany

${ }^{25}$ Department of Pediatric Hematology and Oncology, University Medical Center Hamburg-Eppendorf, Hamburg, Germany

${ }^{26}$ Research Institute Children's Cancer Center Hamburg, Hamburg, Germany

${ }^{27}$ Paul-Flechsig Institute of Neuropathology, University Hospital and Faculty of Medicine, Leipzig, Germany

${ }^{28}$ Institute of Neuropathology, University of Giessen, Giessen, Germany

${ }^{29}$ Frankfurt Cancer Institute (FCl), University Hospital, Goethe University Frankfurt am Main, Frankfurt am Main, Germany

${ }^{30}$ Institute of Neurology (Edinger-Institute), University Hospital, Goethe University Frankfurt am Main, Frankfurt am Main, Germany

${ }^{31}$ German Cancer Consortium (DKTK), Partner site Frankfurt/ Mainz, Frankfurt am Main, Germany

${ }^{32}$ German Cancer Research Center (DKFZ), Heidelberg, Germany

${ }^{33}$ Department of Neuropathology, Otto-von-Guericke University, Magdeburg, Germany

${ }^{34}$ Department of Neuropathology, University of Tübingen, Tübingen, Germany

${ }^{35}$ Division of Neuropathology, National Hospital for Neurology and Neurosurgery, University College London Hospitals NHS Foundation Trust, Queen Square, London, UK

${ }^{36}$ Department of Neurodegenerative Disease, UCL Queen Square Institute of Neurology, Queen Square, London, UK

${ }^{37}$ Department of Pathology, NYU Langone Medical Center, New York, NY, USA

${ }^{38}$ Laboratory of Pathology, Center for Cancer Research, National Cancer Institute, National Institutes of Health, Bethesda, MD, USA

${ }^{39}$ Neuro-Oncology Branch, National Cancer Institute, Bethesda, MD, USA

${ }^{40}$ Department of Pathology, St. Jude Children's Research Hospital, Memphis, TN, USA

${ }^{41}$ Division of Brain Tumor Translational Research, National Cancer Center Research Institute, Chuo-ku, Tokyo, Japan

${ }^{42}$ Institute of Neuropathology, Heinrich Heine University, Düsseldorf, Germany

${ }^{43}$ German Cancer Consortium (DKTK), Partner Site Essen/Düsseldorf, Germany

${ }^{44}$ Children's Brain Tumour Research Centre, University of Nottingham, Nottingham, UK

${ }^{45}$ Department of Human Genetics, McGill University, Montreal, QC H3A 1B1, Canada

${ }^{46}$ Department of Pediatrics, McGill University, Montreal, QC H4A 3J1, Canada

${ }^{47}$ The Research Institute of the McGill University Health Center, Montreal, QC H4A 3J1, Canada

${ }^{48}$ Prague Brain Tumor Research Group, Second Faculty of Medicine, Charles University and University Hospital Motol, Prague, Czech Republic

${ }^{49}$ Department of Pathology and Molecular Medicine, Second Faculty of Medicine, Charles University and University Hospital Motol, Prague, Czech Republic

${ }^{50}$ Department of Pediatric Haematology and Oncology, Second Faculty of Medicine, Charles University and University Hospital Motol, Prague, Czech Republic

${ }^{51}$ Department of Pathology, Amsterdam University Medical Centers, Location VUmc and Brain Tumor Center Amsterdam, Amsterdam, The Netherlands

${ }^{52}$ Clinical Cooperation Unit Neurooncology, German Consortium for Translational Cancer Research (DKTK), German Cancer Research Center

(DKFZ), Heidelberg, Germany

${ }^{53}$ Department of Neurology and Neurooncology Program, National Center for Tumor Diseases, Heidelberg University Hospital, Heidelberg, Germany

\# indicates shared authorship

* Corresponding authors 
bioRxiv preprint doi: https://doi.org/10.1101/2021.04.23.441059; this version posted April 23, 2021. The copyright holder for this preprint (which was not certified by peer review) is the author/funder. All rights reserved. No reuse allowed without permission.

Corresponding authors:

Felix Sahm, MD, PhD

Department of Neuropathology

University Hospital Heidelberg

and

Clinical Cooperation Unit Neuropathology (B300)

German Cancer Research Center (DKFZ)

Im Neuenheimer Feld 224

69120 Heidelberg, Germany

Fon: +49-6221 56-37886

felix.sahm@med.uni-heidelberg.de

Kristian W. Pajtler, MD

Hopp Children's Cancer Center Heidelberg (KiTZ)

and

Division of Pediatric Neurooncology,

German Cancer Research Center (DKFZ)

and

Department of Pediatric Oncology, Hematology, Immunology and Pulmonology

University Hospital Heidelberg

Im Neuenheimer Feld 430

69120 Heidelberg, Germany

Fon: +49-6221 42-4585

k.pajtler@kitz-heidelberg.de 


\begin{abstract}
Ependymomas encompass a heterogeneous group of central nervous system (CNS) neoplasms that occur along the entire neuroaxis. In recent years, extensive (epi-)genomic profiling efforts have identified several molecular groups of ependymoma that are characterized by distinct molecular alterations and/or patterns. Based on unsupervised visualization of a large cohort of genome-wide DNA methylation data, we identified a highly distinct group of pediatric-type tumors $(n=40)$ forming a cluster separate from all established CNS tumor types, of which a high proportion were histopathologically diagnosed as ependymoma. RNA sequencing revealed recurrent fusions involving the pleomorphic adenoma gene-like 1 (PLAGL1) gene in 19 of 20 of the samples analyzed, with the most common fusion being EWSR1:PLAGL1 $(\mathrm{n}=13)$. Five tumors showed a PLAGL1:FOXO1 fusion and one a PLAGL1:EP300 fusion. High transcript levels of PLAGL1 were noted in these tumors, with concurrent overexpression of the imprinted genes H19 and IGF2, which are regulated by PLAGL1. Histopathological review of cases with sufficient material $(n=16)$ demonstrated a broad morphological spectrum of largely ependymoma-like tumors. Immunohistochemically, tumors were GFAP-positive and OLIG2- and SOX10-negative. In 3/16 of the cases, a dot-like positivity for EMA was detected. Consistent with other fusion-positive ependymal groups, all tumors in our series were located in the supratentorial compartment. Median age of the patients at the time of diagnosis was 6.2 years. Analysis of time to progression or recurrence revealed survival times comparable to those of patients with ZFTA:RELA-fused ependymoma. In summary, our findings suggest the existence of a novel group of supratentorial ependymomas that are characterized by recurrent PLAGL 1 fusions and enriched for pediatric patients.
\end{abstract}

Keywords: Ependymoma; Supratentorial; PLAGL1; EWSR1; FOXO1; EP300; Gene fusion 


\section{Introduction}

Ependymomas encompass a heterogeneous group of central nervous system (CNS) neoplasms that occur along the entire neuroaxis and can affect both children and adults ${ }^{1}$. DNA methylation and gene expression profiling efforts in recent years have identified several molecular groups of ependymoma across different anatomic sites of the CNS with distinct clinicopathological characteristics and molecular alterations or patterns ${ }^{2-10}$. Within the supratentorial compartment, two molecularly defined types of ependymoma are characterized by recurrent gene fusions, one involving the gene ZFTA (formerly referred to as C11orf95, most frequently fused to $R E L A$ ), and the other involving $Y A P 1^{2,5}$. More recently, several reports have expanded on the spectrum of gene fusions observed in supratentorial ependymoma and ependymoma-like tumors, in particular in the pediatric setting 11-13. Implementing these molecular markers into the WHO classification for brain tumors is of paramount importance in overcoming the challenges of histologically diverse tumor types and in increasing diagnostic accuracy. Still, many cases do not fit into the as of yet established CNS tumor types, leaving clinicians and patients with unclear or even incorrect diagnoses in further decision making.

Genome-wide DNA methylation profiling has emerged as a powerful tool for both robust classification of known CNS tumor entities and identification of novel and clinically relevant subclasses of brain tumors with characteristic alterations ${ }^{2,14}$. Here, we describe a molecularly distinct subset of supratentorial neoplasms $(n=40)$ with ependymal appearance identified by investigation of a large cohort of DNA methylation data. These tumors harbor recurrent fusions involving the pleomorphic adenoma gene-like 1 (PLAGL1) gene. 


\section{Materials and methods}

\section{Sample collection}

Tumor samples and retrospective clinical data from 40 patients were obtained from multiple national and international collaborating centers and collected at the Department of Neuropathology of the University Hospital Heidelberg (Germany). Sample selection was based on unsupervised visualization of genome-wide DNA methylation data that revealed a molecularly distinct group of tumors $(n=40)$ forming a cluster separate from all established entities. A proportion of data was generated in the context of the Molecular Neuropathology 2.0 study. Analysis of tissue and clinical data was performed in accordance with local ethics regulations. Clinical details of the patients are listed in Supplementary Table 1 (online resource).

\section{Histology and immunohistochemistry}

For all cases with sufficient material $(n=16)$, histological review of an H\&E-stained slide was performed according to the World Health Organization (WHO) 2016 classification of tumors of the CNS ${ }^{15}$. Immunohistochemical staining was performed on a Ventana BenchMark ULTRA Immunostainer using the ultraView Universal DAB Detection Kit (Ventana Medical Systems, Tucson, AZ, USA). Antibodies were directed against: glial fibrillary acid protein (GFAP; Z0334, rabbit polyclonal, 1:1000 dilution, Dako Agilent, Santa Clara, CA, USA), epithelial membrane antigen (EMA; clone GP1.4, mouse monoclonal, dilution 1:1000, Thermo Fisher Scientific, Fremont, CA, USA), Sry-related HMG-BOX gene 10 (SOX10; clone EP268, rabbit monoclonal, dilution 1:100, Cell Marque Corp., Rocklin, CA, USA) and oligodendrocyte lineage transcription factor 2 (OLIG2; clone EPR2673, rabbit monoclonal, dilution 1:50, Abcam, Cambridge, UK).

\section{DNA methylation array processing and copy number profiling}

Genome-wide DNA methylation profiling of all samples was performed using the Infinium MethylationEPIC (EPIC) BeadChip (Illumina, San Diego, CA, USA) or Infinium HumanMethylation450 (450k) BeadChip array (Illumina) according to the manufacturer's instructions and as previously described ${ }^{14}$. Raw data were generated at the Department of Neuropathology of the University Hospital Heidelberg, the Genomics and Proteomics Core Facility of the German Cancer Research Center (DKFZ) or at respective international collaborator institutes, using both fresh-frozen and formalin-fixed paraffin-embedded (FFPE) tissue samples. All computational analyses were performed in $\mathrm{R}$ version 3.6.0 (R Development Core Team, 2016; https://www.R-project.org). Copy-number variation analysis from 450k and EPIC methylation array data was performed using the conumee Bioconductor package version 1.12.0 ${ }^{16}$. Raw signal intensities were obtained from IDAT-files using the minfi Bioconductor package version 1.21.4. Illumina EPIC and 450k samples were merged to a combined data set by selecting the intersection of probes present on both arrays (combineArrays function, minfi). Each sample was individually normalized by performing a background correction (shifting of the $5 \%$ percentile of negative control probe intensities to 0 ) and a dye-bias correction (scaling of the mean of normalization control probe intensities to 10,000) for both color channels. Subsequently, a correction for the array type (450k/EPIC) was performed by fitting univariable, linear models to the log2-transformed intensity values (removeBatchEffect 
function, limma package version 3.30.11). The methylated and unmethylated signals were corrected individually. Beta-values were calculated from the retransformed intensities using an offset of 100 (as recommended by Illumina). All samples were checked for duplicates by pairwise correlation of the genotyping probes on the 450k/EPIC array. To perform unsupervised non-linear dimension reduction, the remaining probes after standard filtering ${ }^{14}$ were used to calculate the 1-variance weighted Pearson correlation between samples. The resulting distance matrix was used as input for t-SNE analysis (t-distributed stochastic neighbor embedding; Rtsne package version 0.13 ). The following non-default parameters were applied: theta $=0$, pca $=F$, max_iter $=10,000$ perplexity $=20$.

\section{RNA sequencing and analysis}

RNA was extracted from FFPE tissue samples using the automated Maxwell system with the Maxwell 16 LEV RNA FFPE Kit (Promega, Madison, WI, USA), according to the manufacturer's instructions. Transcriptome analysis using messenger RNA (mRNA) sequencing of samples for which RNA of sufficient quality and quantity was available was performed on a NextSeq 500 instrument (Illumina) as previously described ${ }^{17}$. This was possible for 20 tumors within the novel group and 14 ZFTA:RELA-fused ependymomas. In addition, a reference cohort of other glioma and glioneuronal subtypes were used for differential gene expression analysis (YAP1:MAMLD1-fused ependymoma $(n=3)$, central neurocytoma $(n=9)$, extraventricular neurocytoma $(n=8)$, dysembryoplastic neuroepithelial tumor $(n=11)$, papillary glioneuronal tumor $(n=9), K I A A 1549: B R A F$-fused pilocytic astrocytoma $(n=14)$, diffuse midline glioma $\mathrm{H} 3$ K27M mutant $(n=14)$ and glioblastoma IDH-wildtype $(n=9))$. Fastq files from transcriptome sequencing were used for de novo annotation of fusion transcripts using the deFuse ${ }^{18}$ and Arriba (v1.2.0) ${ }^{19}$ algorithms with standard parameters. All further analysis was performed in $\mathrm{R}$ (version 3.6.0; R Core Team, 2019) using the DESeq2 package (v1.28.1) ${ }^{20}$. Principal Component Analysis (PCA) was performed after variance stabilizing transformation of the count data and normalization with respect to library size, based on the selection of the top 1,000 most variable genes with relative log expression normalization. Similarities between samples were determined by computing Manhattan distances on the variance stabilized data followed by unsupervised hierarchical clustering. Differential expression testing was performed on raw count data after fitting a negative binomial model. P-values were adjusted for multiplicity by applying the Benjamini-Hochberg correction.

\section{Targeted next generation DNA sequencing and mutational analysis}

Genomic DNA was extracted from FFPE tumor tissue samples of 18 patients within the cohort using the automated Maxwell system with the Maxwell 16 FFPE Plus LEV DNA Purification Kit (Promega, Madison, WI, USA), according to the manufacturer's instructions. Capture-based next-generation DNA sequencing was performed on a NextSeq 500 instrument (Illumina) as previously described ${ }^{21}$ using a custom brain tumor panel (Agilent Technologies, Santa Clara, CA, USA) covering the entire coding and selected intronic and promoter regions of 130 genes of particular relevance in CNS tumors (Supplementary Table 2, online resource).

\section{Statistical analysis}


Statistical analysis was performed using GraphPad Prism 9 (GraphPad Software, La Jolla, CA, USA). Data on survival could be retrospectively retrieved for ten patients. Distribution of time to progression or recurrence (TTP) after surgery was estimated by the Kaplan-Meier method and compared between groups with the log-rank test. Patients lost to follow-up are censored at date of last contact in analysis of TTP. P-values below 0.05 were considered significant. 


\section{Results}

\section{DNA methylation profiling reveals a molecular distinct group of ependymoma}

DNA methylation profiling has emerged as a powerful approach for robust classification of CNS neoplasms ${ }^{14}$. Using a screening approach based on unsupervised visualization of a large cohort of genome-wide DNA methylation data, we identified a highly distinct group of tumors $(n=40)$ forming a cluster separate from all established entities of which a high proportion of tumors (19/32, 59\%) were histopathologically diagnosed as ependymoma. A more focused $t-$ SNE analysis of DNA methylation patterns of these samples alongside 453 other wellcharacterized ependymal neoplasms (reference samples included in the current version of the Heidelberg DNA methylation classifier with a calibrated score $>0.9$ ) confirmed the distinct nature of this novel group (Fig. 1 and Supplementary Figure 1, online resource). Analysis of copy number variations (CNVs) derived from DNA methylation array data revealed a relatively balanced profile in most of the cases, with structural aberrations on chromosome 22q (21/40, $52.5 \%)$ and $6 q(19 / 40,47.5 \%)$ most frequently observed (Supplementary Figure 2a, online resource). A chromothripsis-like pattern affecting chromosomes 6 and 13 was seen in one of the samples (Supplementary Figure 2b, online resource). In one case a homozygous deletion of $C D K N 2 A / B$ was detected. An integrated plot of $C N V$ s identified in all samples is given in Supplementary Figure 2c (online resource).

\section{Recurrent rearrangements involving PLAGL1 are characteristic for the novel group of ependymoma}

Since a high proportion of supratentorial ependymomas are driven by gene fusions involving ZFTA (C11orf95, most frequently fused to RELA) or YAP1, we performed mRNA sequencing of all samples with sufficient material $(n=20)$. In 19/20 of the cases, a gene fusion involving PLAGL1 was detected, conserving the zinc finger structure (C2H2 type) as part of the fusion product, with either EWSR1 as 5' partner or FOXO1 or EP300 as a 3' partner (Fig. 2a-c). In the most common EWSR1:PLAGL1 fusions $(\mathrm{n}=13)$, exons $1-9$ or $1-8$ of EWSR1 (NM_013986), which is located on chromosome 22q12.2, were fused to exon 5 of PLAGL1 (NM_001289039), which is found on chromosome 6q24.2. Five out of 20 cases were observed with exons 1-5 of PLAGL1 fused to FOXO1 upstream of exons 2-3 (NM_0017612) were also observed. In one case, exons 1-5 of PLAGL1 are fused to exons 15-31 of EP300 (NM_001429). In all rearrangements, the DNA binding domain (zinc finger structure) of PLAGL1 was retained and fused to the respective transactivation domain (TAD) of the partner gene (Fig. 2a-C). We next performed an exploratory differential gene expression analysis of tumor samples $(n=20)$ within the novel group in comparison to ZFTA:RELA-fused ependymomas $(n=14)$. Unsupervised hierarchical clustering demonstrated a clear segregation of tumor samples in comparison to ZFTA:RELA-fused ependymoma (Fig. $2 \mathrm{~d}$ ). These results were recapitulated by PCA of normalized transcript counts (Fig. 2e). Quantification of mRNA expression revealed that the PLAGL1 gene itself was more highly expressed in tumors within the novel group than in ZFTA:RELA-fused ependymoma (adjusted $\mathrm{p}=1.22 \mathrm{e}-14$; Fig. $2 \mathrm{f}$ and g). Additionally, upregulated genes of potential interest included $\mathrm{H} 19$ and IGF2 (adjusted $\mathrm{p}=1.31 \mathrm{e}-83$, adjusted $\mathrm{p}=5.04 \mathrm{e}-08$; Fig. $2 \mathrm{~h}$ and $\mathrm{i}$ ), both regulated by $P L A G L 1$ and with known functions in the tumorigenesis of different cancers ${ }^{22}$. RELA and ZFTA transcript levels were upregulated in ZFTA:RELA-fused ependymomas (adjusted $p=1.03 \mathrm{e}-61$ and adjusted $p=1.10 \mathrm{e}-19$, respectively; Fig. $2 \mathrm{j}$ and k). Differential gene expression analysis 
between tumors within the novel group and a reference cohort of other glial and glioneuronal subtypes confirmed high transcript levels of PLAGL1 (adjusted $p=2.35 \mathrm{e}-18$ ), H19 (adjusted $p$ $=9.12 \mathrm{e}-15$ ), IGF2 (adjusted $\mathrm{p}=7.91 \mathrm{e}-06$ ) and DLK1 (adjusted $\mathrm{p}=1.12 \mathrm{e}-10$ ) in the PLAGL1fused cohort (Figure 3a-c and Supplementary Figure 3, online resource). Expression of particular markers differentially expressed in astrocytic and in ependymal neoplasms ${ }^{23-25}$ revealed low OLIG2 and SOX10 expression (adjusted $p=3.89 \mathrm{e}-26$ and adjusted $p=7.75 \mathrm{e}-$ 65 ) within the novel group, with similar expression of GFAP (Figure 3d-f and Supplementary Figure 3, online resource). Analysis of the mutational landscape of 19/40 tumors in the novel group using targeted next-generation sequencing revealed TERT promoter mutations (C228T) in two of the cases (Supplementary Table 1, online resource), with no other relevant events involving putative brain tumor genes.

\section{Clinical characteristics and morphological features demonstrate pediatric-type tumors with ependymoma-like appearance}

Analysis of available clinical data demonstrated that median age of the patients at the time of diagnosis was 6.2 years $(n=25$; range $0-30$; with $92 \%$ of the tumors occurring in patients $<17$ years of age, Fig. 4a) and the sex distribution was relatively balanced ( $F / M=1: 1.2$, Fig. 4b). All tumors in our series were located supratentorially (Fig. 4c). Outcome data were available for 11 patients. Analysis of time to recurrence in comparison to ZFTA:RELA-fused ependymomas $(n=80)$ revealed a similar outcome $(p=0.18$; Figure $4 d)$. The initial histopathological diagnoses of the tumors within the cohort were relatively wide, although a high proportion of cases were designated as ependymoma (19/32, 59\%). Other recurrent diagnoses included 'embryonal tumor' and different low- and high-grade gliomas (Supplementary Table 1, online resource). More detailed descriptions of the cases are given in Supplementary Table 1. A histopathological review of samples with available material $(n=$ 16) confirmed a relatively wide morphological spectrum of tumors with ependymoma-like features (Fig. 5a-i). Histologically, all reviewed tumors shared a moderate to high increase in cellular density in a mostly fine neurofibrillary matrix with prominent microcystic changes (Fig. 5a-d). The tumor cells typically had monomorphic, round to oval nuclei with finely dispersed chromatin and prominent nucleoli. Single cases presented more pleomorphic cells. In many cases, perivascular pseudorosettes were observed, at least focally. Two of the cases showed focal oligodendroglial morphology with perinuclear halos due to cytoplasmatic clearing (Fig. $5 e)$. Extensive calcification was seen in a small number of tumors $(n=3)$. Necrosis was not observed. Mitotic activity was generally low, with exception of two cases. Immunoreactivity for GFAP was present in all cases (Fig. 5f). The tumor cells neither expressed OLIG2 nor SOX10 (Fig. $5 g$ and h). In 3/16 of the cases, a dot-like positivity for EMA was detected (Fig. 5i). 


\section{Discussion}

Here, we provide evidence for the pathobiological heterogeneity of ependymal tumors beyond the established spectrum by reporting the existence of an epigenetically distinct group of rare pediatric-type supratentorial neoplasms with often ependymoma-like appearance that shows recurrent gene fusions involving the PLAGL1 gene.

Our findings suggest rearrangements involving PLAGL1, particularly EWSR1:PLAGL1 and PLAGL1:FOXO1 fusions, as a molecular hallmark of this novel group of tumors. Gene fusions of PLAGL1 with EWSR1 have been reported exceptionally rarely in neoplasms of the CNS, including single cases of a SMARCB1-deficient atypical teratoid/rhabdoid tumor (AT/RT) ${ }^{26}$ and a glioneuronal tumor, not elsewhere classified (NEC) ${ }^{27}$. However, in a very recent report, a PLAGL1:EWSR1 fusion was described in a supratentorial ependymoma of a six-year-old child 11. While EWSR1 has long been known to be involved in gene fusions in Ewing sarcoma and several other tumor entities ${ }^{28}$, the role of $P L A G L 1$ in tumorigenesis is not yet fully understood. The PLAGL1 gene encodes a $\mathrm{C} 2 \mathrm{H} 2$ zinc finger protein that acts as a transcription factor as well as cofactor of other regulatory proteins, and is expressed in diverse types of human tissues amongst others in neural stem/progenitor cells and developing neuroepithelial cells 29,30 . Although its specific role in tumorigenesis is controversial and its functions appear to depend on the cellular context, altered expression of PLAGL1 has been linked to various types of cancer ${ }^{31-33}$. More recent studies provide evidence for its oncogenic function in brain tumors with overexpression of PLAGL1 being involved in tumorigenesis of glioblastoma ${ }^{34,35}$ and interaction of PLAGL family transcription factors in ZFTA:RELA-fused supratentorial ependymoma ${ }^{36}$.

In the EWSR1:PLAGL1 fusions described here, the whole N-terminal transcriptional activation domain (TAD) of EWSR1 is fused in-frame to the zinc finger domain (with DNA binding activity) of PLAGL1, very similar to other oncogenic EWSR1 fusions, in particular rearrangements between EWSR1 and PATZ1 ${ }^{37,38}$. This indicates aberrant recruitment of the TAD of EWSR1 to the DNA binding domain of PLAGL1 with subsequent downstream effects, as described for other EWSR1 rearrangements, as the likely oncogenic function of this fusion ${ }^{39}$. This also fits to the increased expression of PLAGL1 in these samples. In addition, five samples harbored a fusion between PLAGL1 and the transcriptional factor FOXO1, which is a known partner in other rearrangements ${ }^{40,41}$. In the PLAGL1:FOXO1 fusion observed here, the DNA binding domain of PLAGL1 is juxtaposed to the C-terminal TAD of FOXO1, which seems quite similar to $P A X 3: F O X 01$ rearrangements as frequently observed in alveolar rhabdomyosarcoma ${ }^{41}$. In a single case, PLAGL1 was fused to EP300, a fusion partner known from 'CNS tumors with $B C O R$ alteration' ${ }^{42}$. Additionally, upregulated genes included $H 19$ and IGF2, both regulated by PLAGL1 and with known functions in tumorigenesis of different cancers ${ }^{22}$. This might indicate a potential downstream effect of the fusion. However, the precise oncogenic mechanism of the EWSR1:PLAGL1, PLAGL1:FOXO1 and PLAGL1:EP300 chimeric proteins remain to be elucidated. Further studies will be needed to reveal the exact role of the fusions in these tumors.

Another important finding was the relatively wide morphological spectrum of tumors within this group. Although most tumors were originally diagnosed as ependymoma, a significant proportion of cases were designated to other entities, including different low- and high-grade tumors. Consistent with that, a histopathological review of cases with sufficient material revealed a morphologically heterogeneous group of tumors often with ependymoma-like features, compatible with the designation as ependymoma. These findings are supported by 
differential gene expression analysis between tumors within the novel group and a reference cohort of other glial and glioneuronal tumors, that revealed low expression levels of OLIG2 and SOX10, both suggested to distinguish astrocytic from ependymal tumors ${ }^{23-25}$. However, the absence of a unifying morphological pattern in this group of tumors underlines the relevance of molecular profiling for precise diagnosis of these CNS neoplasms. This group has not been identified as a distinct subset in previous large-scale ependymoma studies due to the relatively small case numbers, broad morphology and lack of routine RNA profiling in previous cohorts, again highlighting the importance of RNA sequencing in standard brain tumor diagnostic. According to the structure of specifying 'essential diagnostic criteria' of the upcoming 5th edition of the WHO classification of CNS tumors, we suggest (a) the specific signature by DNA methylation profiling or (b) the combination GFAP expression and PLAGL1 fusions as essential diagnostic criteria for these tumors.

A limitation of our study is the relatively low extent of clinical data, in particular patient outcome data, which allows only a rough estimation of the malignancy of the tumors within this novel group. Considering the high number of cases without sequencing data, it seems also possible that other alterations apart from the described fusions could be present, particularly in those tumors which do not show indication for a PLAGL1 fusion in the copy-number profile. Followup analyses are needed to characterize this new group of CNS neoplasms in more detail.

In summary, we provide new insights into the tumorigenesis of ependymoma and identify PLAGL1 as a putative relevant driver in this entity. Given their ependymoma-like appearance and expression pattern with a high frequency of PLAGL1 fusions, we suggest the term 'supratentorial ependymoma with PLAGL1 fusion' to describe this novel group of tumors. These findings have immediate implications for brain tumor profiling in order to avoid incorrect diagnoses due to lack of alignment with established tumor types. PLAGL1-fusion positive ependymomas should thus be included into upcoming classifications of brain tumors.

Acknowledgements For excellent technical support we sincerely thank the Microarray Unit of the German Cancer Research Center (DKFZ) Genomics and Proteomics Core Facility, as well as I. Leis and M. Schalles (Department of Neuropathology, Institute of Pathology, University Hospital Heidelberg, Heidelberg, Germany). We also thank the German Childhood Cancer Foundation for funding ("Neuropath 2.0 - Increasing diagnostic accuracy in paediatric neurooncology" (DKS 2015.01)). This study was supported by the Hertie Network of Excellence in Clinical Neuroscience. P. Sievers is a fellow of the Hertie Academy of Excellence in Clinical Neuroscience. S. C. Henneken received scholarships of the Mildred-Scheel doctoral program of the German Cancer Aid and the German Academic Scholarship Foundation. S. Brandner was partly funded by the National Institute of Health Research (NIHR) UCLH/UCL Biomedical Research Centre. A subset of the human tissue was obtained from University College London NHS Foundation Trust as part of the UK Brain Archive Information Network (BRAIN UK, Ref: 19/001) which is funded by the Medical Research Council and Brain Tumour Research UK. U. Schüller was supported by the Fördergemeinschaft Kinderkrebszentrum Hamburg.

Author contributions P.S., S.C.H., K.W.P. and F.S. designed the study; P.S., S.C.H., D.T.W.J., K.W.P. and F.S. conducted experiments; P.S., S.C.H., C.B., D.Sc., D.St., K.O., D.T.W.J., K.W.P. and F.S. analyzed data; all authors assisted with material and data collection; all authors contributed to manuscript writing.

Competing interests The authors declare no competing interests. 


\section{References}

1 Louis DN, O. H., Wiestler OD, Cavenee WK. WHO classification of tumours of the central nervous system. Vol. Revised 4 edn. (IARC, Lyon, 2016).

2 Pajtler, K. W. et al. Molecular Classification of Ependymal Tumors across All CNS Compartments, Histopathological Grades, and Age Groups. Cancer Cell 27, 728-743, doi:10.1016/j.ccell.2015.04.002 (2015).

3 Pajtler, K. W. et al. Molecular heterogeneity and CXorf67 alterations in posterior fossa group A (PFA) ependymomas. Acta Neuropathol 136, 211-226, doi:10.1007/s00401-018-1877-0 (2018).

4 Panwalkar, P. et al. Immunohistochemical analysis of H3K27me3 demonstrates global reduction in group-A childhood posterior fossa ependymoma and is a powerful predictor of outcome. Acta Neuropathol 134, 705-714, doi:10.1007/s00401-017-1752-4 (2017).

5 Parker, M. et al. C11orf95-RELA fusions drive oncogenic NF-kappaB signalling in ependymoma. Nature 506, 451-455, doi:10.1038/nature13109 (2014).

6 Ghasemi, D. R. et al. MYCN amplification drives an aggressive form of spinal ependymoma. Acta Neuropathol 138, 1075-1089, doi:10.1007/s00401-019-02056-2 (2019).

7 Cavalli, F. M. G. et al. Heterogeneity within the PF-EPN-B ependymoma subgroup. Acta Neuropathol 136, 227-237, doi:10.1007/s00401-018-1888-x (2018).

8 Witt, H. et al. Delineation of two clinically and molecularly distinct subgroups of posterior fossa ependymoma. Cancer Cell 20, 143-157, doi:10.1016/j.ccr.2011.07.007 (2011).

9 Witt, H. et al. DNA methylation-based classification of ependymomas in adulthood: implications for diagnosis and treatment. Neuro Oncol 20, 1616-1624, doi:10.1093/neuonc/noy118 (2018).

10 Wani, K. et al. A prognostic gene expression signature in infratentorial ependymoma. Acta Neuropathol 123, 727-738, doi:10.1007/s00401-012-0941-4 (2012).

11 Zschernack, V. et al. Supratentorial ependymoma in childhood: more than just RELA or YAP. Acta Neuropathol, doi:10.1007/s00401-020-02260-5 (2021).

12 Pages, M. et al. Diagnostics of pediatric supratentorial RELA ependymomas: integration of information from histopathology, genetics, DNA methylation and imaging. Brain Pathol 29, 325-335, doi:10.1111/bpa.12664 (2019).

13 Tomomasa, R. et al. Ependymoma-like tumor with mesenchymal differentiation harboring C11orf95-NCOA1/2 or -RELA fusion: A hitherto unclassified tumor related to ependymoma. Brain Pathol, e12943, doi:10.1111/bpa.12943 (2021).

14 Capper, D. et al. DNA methylation-based classification of central nervous system tumours. Nature 555, 469-474, doi:10.1038/nature26000 (2018).

15 Louis, D. N. et al. The 2016 World Health Organization Classification of Tumors of the Central Nervous System: a summary. Acta Neuropathol 131, 803-820, doi:10.1007/s00401-016-15451 (2016).

16 Aryee, M. J. et al. Minfi: a flexible and comprehensive Bioconductor package for the analysis of Infinium DNA methylation microarrays. Bioinformatics 30, 1363-1369, doi:10.1093/bioinformatics/btu049 (2014).

17 Stichel, D. et al. Routine RNA sequencing of formalin-fixed paraffin-embedded specimens in neuropathology diagnostics identifies diagnostically and therapeutically relevant gene fusions. Acta Neuropathol 138, 827-835, doi:10.1007/s00401-019-02039-3 (2019).

18 McPherson, A. et al. deFuse: an algorithm for gene fusion discovery in tumor RNA-Seq data. PLoS Comput Biol 7, e1001138, doi:10.1371/journal.pcbi.1001138 (2011).

19 Uhrig, S. et al. Accurate and efficient detection of gene fusions from RNA sequencing data. Genome Res 31, 448-460, doi:10.1101/gr.257246.119 (2021).

20 Love, M. I., Huber, W. \& Anders, S. Moderated estimation of fold change and dispersion for RNA-seq data with DESeq2. Genome Biol 15, 550, doi:10.1186/s13059-014-0550-8 (2014). 
21 Sahm, F. et al. Next-generation sequencing in routine brain tumor diagnostics enables an integrated diagnosis and identifies actionable targets. Acta Neuropathol 131, 903-910, doi:10.1007/s00401-015-1519-8 (2016).

22 Varrault, A. et al. Zac1 regulates an imprinted gene network critically involved in the control of embryonic growth. Dev Cell 11, 711-722, doi:10.1016/j.devcel.2006.09.003 (2006).

23 Kleinschmidt-DeMasters, B. K. et al. SOX10 Distinguishes Pilocytic and Pilomyxoid Astrocytomas From Ependymomas but Shows No Differences in Expression Level in Ependymomas From Infants Versus Older Children or Among Molecular Subgroups. J Neuropathol Exp Neurol 75, 295-298, doi:10.1093/jnen/nlw010 (2016).

24 Ligon, K. L. et al. The oligodendroglial lineage marker OLIG2 is universally expressed in diffuse gliomas. J Neuropathol Exp Neurol 63, 499-509, doi:10.1093/jnen/63.5.499 (2004).

25 Otero, J. J., Rowitch, D. \& Vandenberg, S. OLIG2 is differentially expressed in pediatric astrocytic and in ependymal neoplasms. J Neurooncol 104, 423-438, doi:10.1007/s11060-0100509-x (2011).

26 Ramkissoon, S. H. et al. Clinical targeted exome-based sequencing in combination with genome-wide copy number profiling: precision medicine analysis of 203 pediatric brain tumors. Neuro Oncol 19, 986-996, doi:10.1093/neuonc/now294 (2017).

27 Lopez-Nunez, O. et al. The spectrum of rare central nervous system (CNS) tumors with EWSR1non-ETS fusions: experience from three pediatric institutions with review of the literature. Brain Pathol 31, 70-83, doi:10.1111/bpa.12900 (2021).

28 Thway, K. \& Fisher, C. Mesenchymal Tumors with EWSR1 Gene Rearrangements. Surg Pathol Clin 12, 165-190, doi:10.1016/j.path.2018.10.007 (2019).

29 Valente, T., Junyent, F. \& Auladell, C. Zac1 is expressed in progenitor/stem cells of the neuroectoderm and mesoderm during embryogenesis: differential phenotype of the Zac1expressing cells during development. Dev Dyn 233, 667-679, doi:10.1002/dvdy.20373 (2005).

30 Vega-Benedetti, A. F. et al. PLAGL1: an important player in diverse pathological processes. J Appl Genet 58, 71-78, doi:10.1007/s13353-016-0355-4 (2017).

31 Abdollahi, A. LOT1 (ZAC1/PLAGL1) and its family members: mechanisms and functions. J Cell Physiol 210, 16-25, doi:10.1002/jcp.20835 (2007).

32 Godlewski, J. et al. PLAGL1 (ZAC1/LOT1) Expression in Clear Cell Renal Cell Carcinoma: Correlations with Disease Progression and Unfavorable Prognosis. Anticancer Res 36, 617-624 (2016).

$33 \mathrm{Su}, \mathrm{H}$. C. et al. Gene expression profiling identifies the role of Zac1 in cervical cancer metastasis. Sci Rep 10, 11837, doi:10.1038/s41598-020-68835-0 (2020).

34 Hide, T. et al. Sox11 prevents tumorigenesis of glioma-initiating cells by inducing neuronal differentiation. Cancer Res 69, 7953-7959, doi:10.1158/0008-5472.CAN-09-2006 (2009).

$35 \mathrm{Li}, \mathrm{C}$. et al. Tumor edge-to-core transition promotes malignancy in primary-to-recurrent glioblastoma progression in a PLAGL1/CD109-mediated mechanism. Neurooncol Adv 2, vdaa163, doi:10.1093/noajnl/vdaa163 (2020).

36 Arabzade, A. et al. ZFTA-RELA Dictates Oncogenic Transcriptional Programs to Drive Aggressive Supratentorial Ependymoma. Cancer Discov, doi:10.1158/2159-8290.CD-20-1066 (2021).

37 Siegfried, A. et al. EWSR1-PATZ1 gene fusion may define a new glioneuronal tumor entity. Brain Pathol 29, 53-62, doi:10.1111/bpa.12619 (2019).

38 Rossi, S. et al. Expanding the spectrum of EWSR1-PATZ1 rearranged CNS tumors: An infantile case with leptomeningeal dissemination. Brain Pathol, e12934, doi:10.1111/bpa.12934 (2020).

39 Krystel-Whittemore, M. et al. Novel and established EWSR1 gene fusions and associations identified by next-generation sequencing and fluorescence in-situ hybridization. Hum Pathol 93, 65-73, doi:10.1016/j.humpath.2019.08.006 (2019).

40 Antonescu, C. R. et al. Novel GATA6-FOXO1 fusions in a subset of epithelioid hemangioma. Mod Pathol, doi:10.1038/s41379-020-00723-4 (2020). 
bioRxiv preprint doi: https://doi.org/10.1101/2021.04.23.441059; this version posted April 23, 2021. The copyright holder for this preprint (which was not certified by peer review) is the author/funder. All rights reserved. No reuse allowed without permission.

41 Linardic, C. M. PAX3-FOXO1 fusion gene in rhabdomyosarcoma. Cancer Lett 270, 10-18, doi:10.1016/j.canlet.2008.03.035 (2008).

42 Tauziede-Espariat, A. et al. The EP300:BCOR fusion extends the genetic alteration spectrum defining the new tumoral entity of "CNS tumors with BCOR internal tandem duplication". Acta Neuropathol Commun 8, 178, doi:10.1186/s40478-020-01064-8 (2020). 
bioRxiv preprint doi: https://doi.org/10.1101/2021.04.23.441059; this version posted April 23, 2021. The copyright holder for this preprint (which was not certified by peer review) is the author/funder. All rights reserved. No reuse allowed without permission.

Fig. 1 DNA methylation profiling reveals a molecular distinct group of ependymoma. t-distributed stochastic neighbor embedding (t-SNE) analysis of DNA methylation profiles of the 40 tumors investigated (EPN_PLAGL1) alongside selected reference samples. Reference DNA methylation classes: ependymoma posterior fossa group $A$ (EPN_PFA), ependymoma posterior fossa group B (EPN_PFB), ependymoma spinal (EPN_SPINE), ependymoma with ZFTA fusion (EPN_ZFTA), ependymoma with YAP1 fusion (EPN_YAP1), myxopapillary ependymoma $\left(E P N \_M P E\right)$, spinal ependymoma (EPN_SPINE), posterior fossa subependymoma (EPN_PF_SE), spinal subependymoma (EPN_SPINE_SE), supratentorial subependymoma (EPN_ST_SE) and spinal ependymoma with $M Y C N$ amplification (EPN_SPINE_MYC). Additional clustering analyses indicated that the PLAGL1 cohort can potentially be further subdivided into two clusters (not shown).

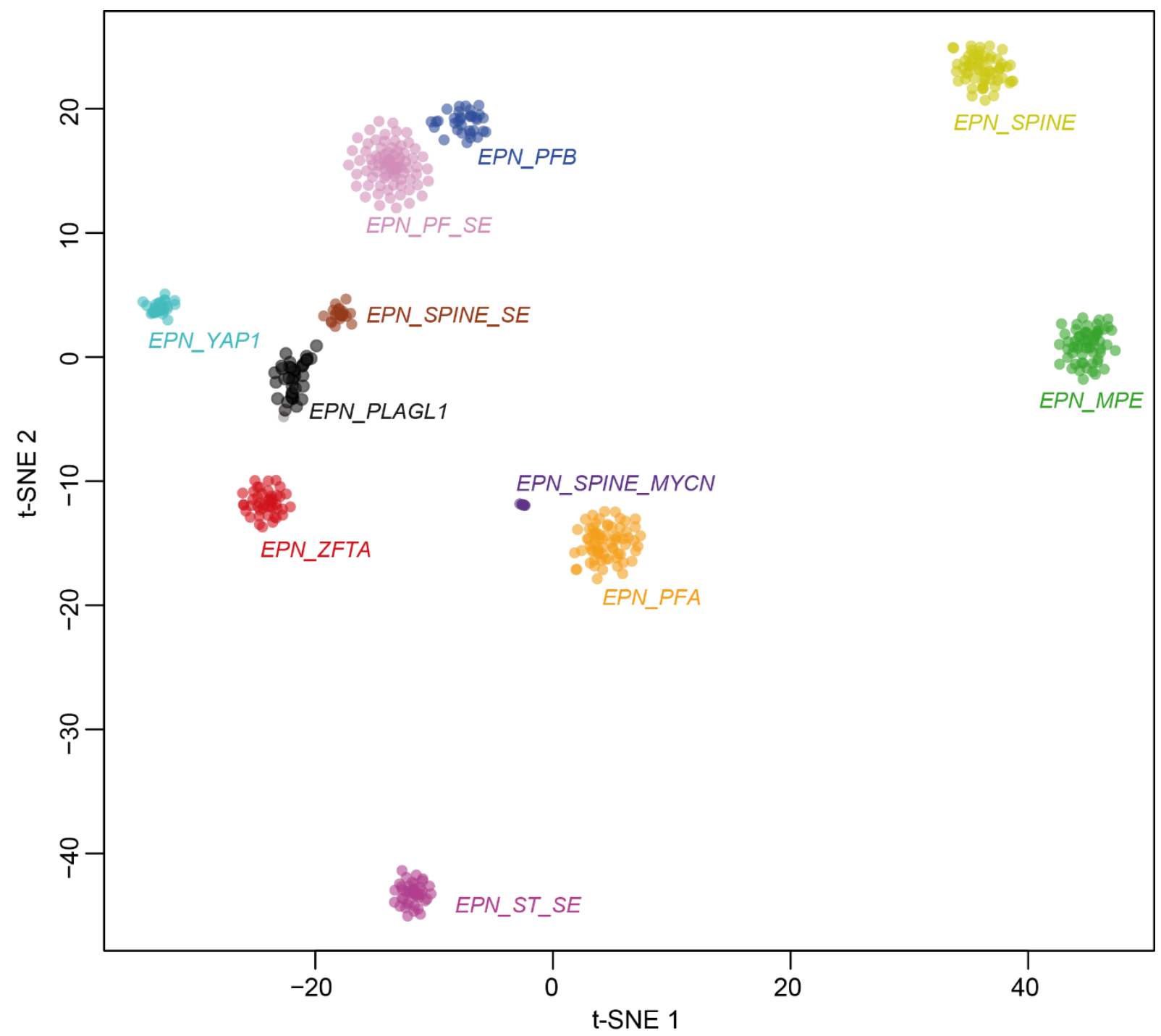


bioRxiv preprint doi: https://doi.org/10.1101/2021.04.23.441059; this version posted April 23, 2021. The copyright holder for this preprint (which was not certified by peer review) is the author/funder. All rights reserved. No reuse allowed without permission.

Fig. 2 Illustration of the PLAGL1 fusion genes and transcriptional profiling of tumors samples in the novel group. Visualization of the PLAGL1 fusion genes detected by RNA sequencing for three selected samples. EWSR1:PLAGL1 fusion in case \#1, in which exons 1-9 of EWSR1, as the 5' partner, are fused to exon 5 of PLAGL1 (a), PLAGL1:FOXO1 fusion in case \#14, in which exons 1-5 of PLAGL1 are fused to exons 2-3 of FOXO1 as the 3' partner (b), and PLAGL1:EP300 fusion in case \#19, in which exons 1-5 of PLAGL1 are fused to exons 15-31 of EP300 as the 3' partner (c), conserving the zinc finger structure ( $\mathrm{C} 2 \mathrm{H} 2$ type) as part of the fusion products. Differences in gene expression profiles between samples in the novel group and ZFTA:RELA-fused ependymomas. Normalized transcript counts from samples in the novel group and ZFTA:RELA-fused ependymomas clustered by Pearson's correlation coefficient (d) and principal component analysis (e). Volcano plot depicting genes differentially expressed between samples in the novel group versus ZFTA:RELA-fused ependymomas (f). PLAGL1 (g), H19 (h), IGF2 (i), ZFTA (j), and RELA (k) expression in the novel group $(\mathrm{n}=20)$ compared to ZFTA:RELA-fused ependymoma samples $(n=14)$.
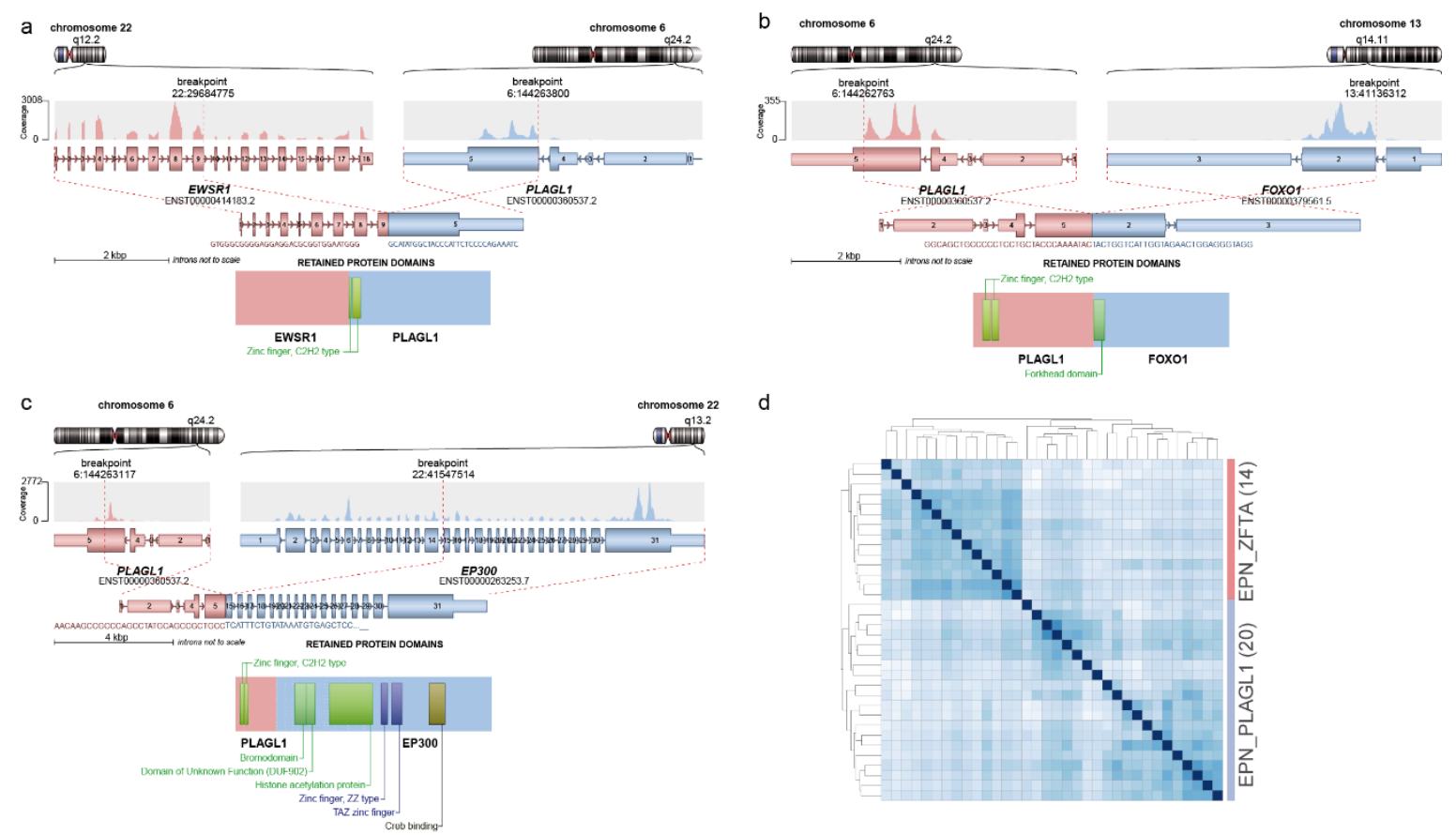

d

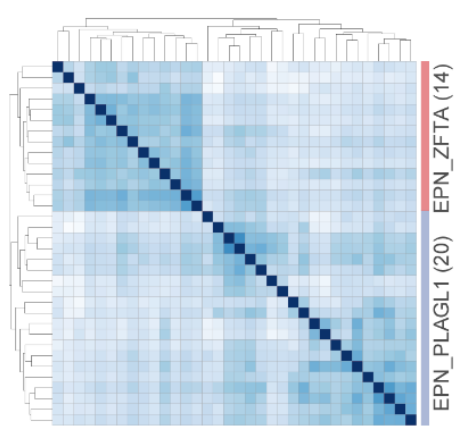

e
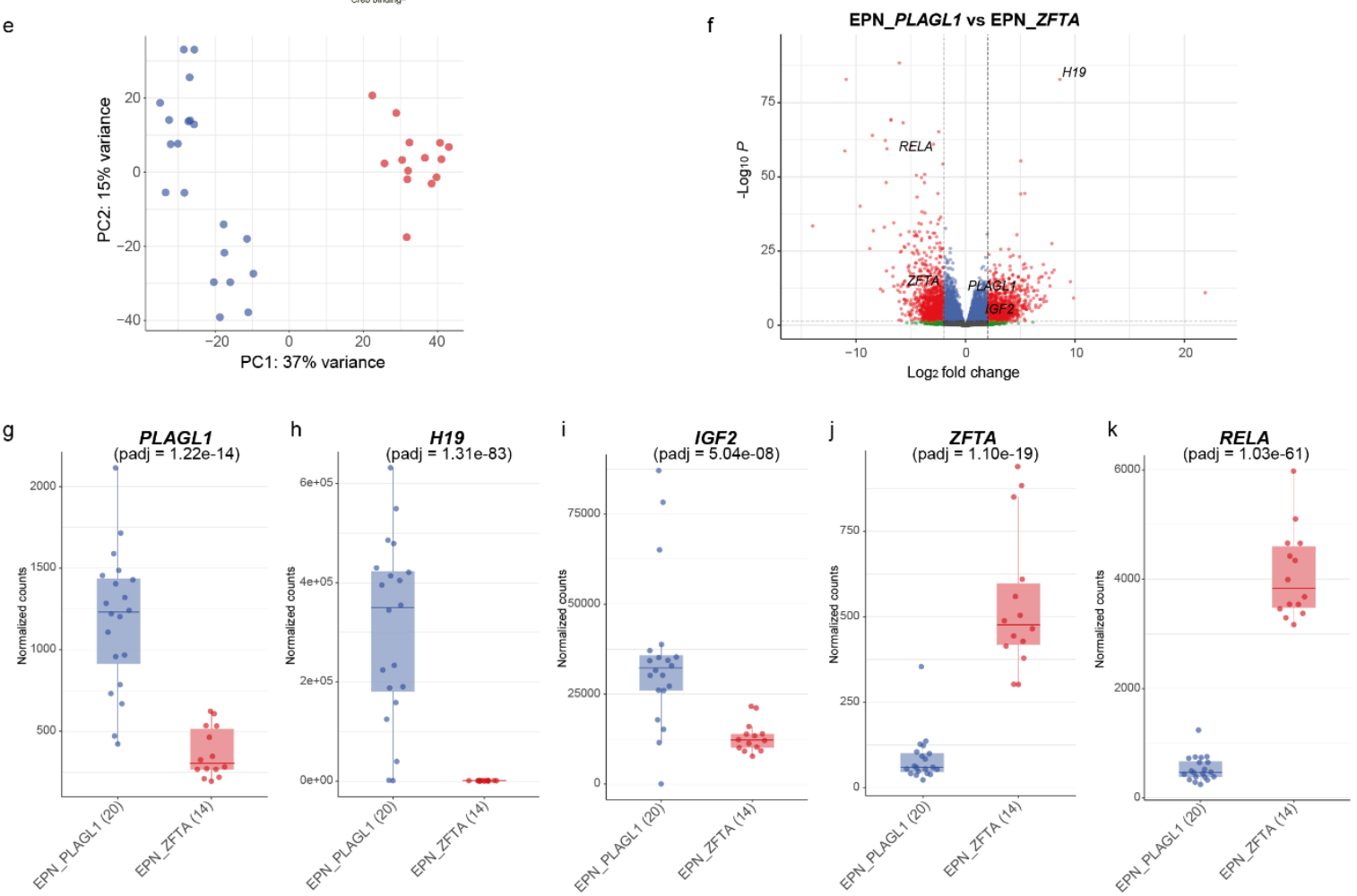
bioRxiv preprint doi: https://doi.org/10.1101/2021.04.23.441059; this version posted April 23, 2021. The copyright holder for this preprint (which was not certified by peer review) is the author/funder. All rights reserved. No reuse allowed without permission.

Fig. 3 Transcriptional profiling of PLAGL1-altered ependymoma. Differential gene expression analysis between samples in the novel group (EPN_PLAGL1) and a reference cohort of different glial/glioneuronal tumors (ZFTA:RELA-fused ependymoma (EPN_ZFTA), YAP1:MAMLD1-fused ependymoma (EPN_YAP1), central neurocytoma $(C N)$, extraventricular neurocytoma $(E V N)$, dysembryoplastic neuroepithelial tumor (DNT), papillary glioneuronal tumor (PGNT), KIAA1549:BRAF-fused pilocytic astrocytoma (PA), diffuse midline glioma H3 K27Mmutant (DMG) and glioblastoma IDH-wildtype (GBM). PLAGL1, IGF2 and H19 are more highly expressed in EPN_PLAGL1 cases when compared with representative glial/glioneuronal tumors (a-c). GFAP levels are similar compared to different glial/glioneuronal tumors (d). Expression of markers differentially expressed in astrocytic and in ependymal tumors revealed low OLIG2 and SOX10 expression in EPN_PLAGL1 compared to astrocytic/glioneuronal tumors (e,f).
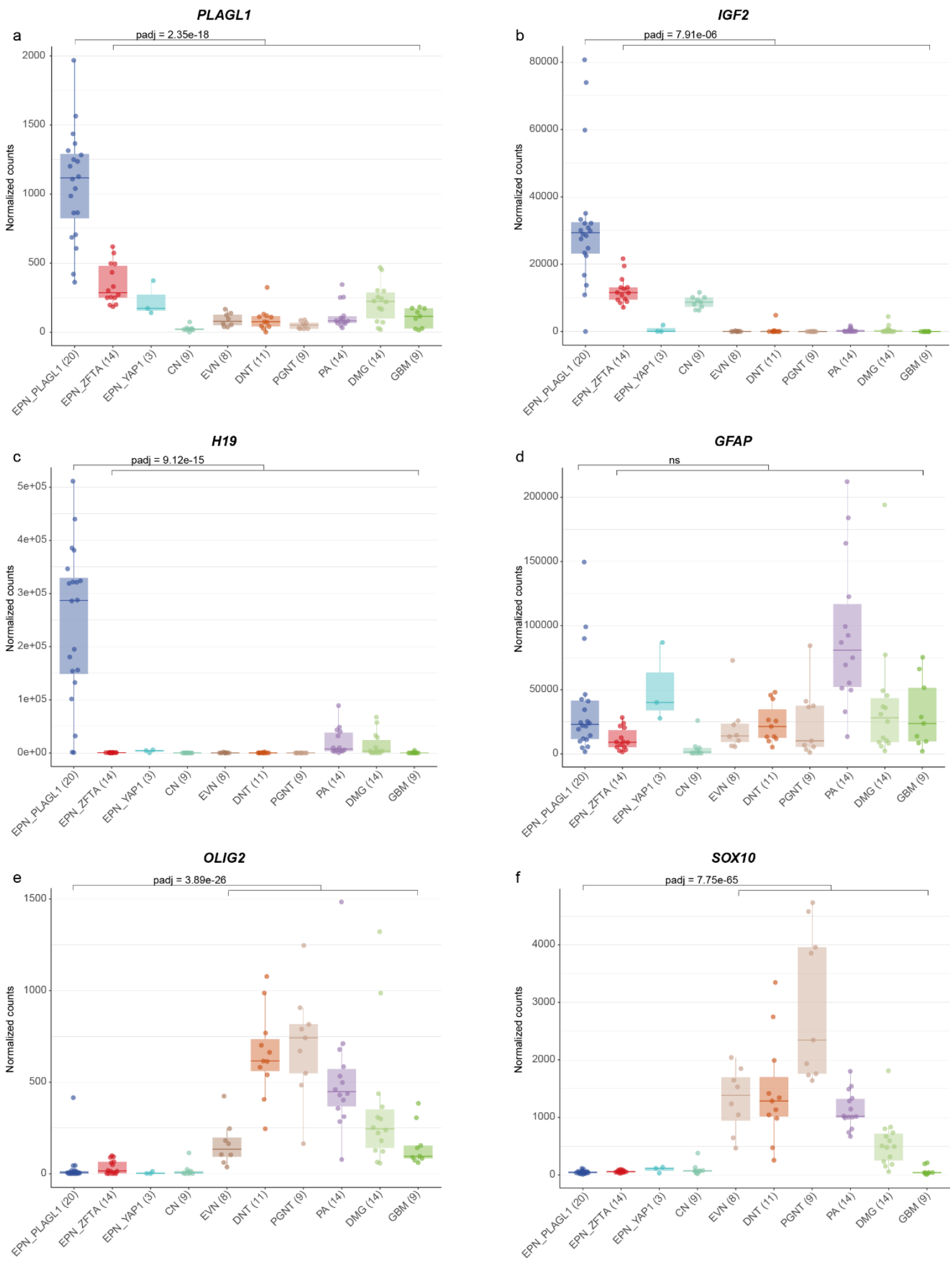
bioRxiv preprint doi: https://doi.org/10.1101/2021.04.23.441059; this version posted April 23, 2021. The copyright holder for this preprint (which was not certified by peer review) is the author/funder. All rights reserved. No reuse allowed without permission.

Fig. 4 Clinical features of the investigated cohort. Age at diagnosis with the median age of 6.2 years (a), patient sex distribution (b) and distribution of tumor location (c). Time to progression or recurrence (TTP) of 11 patients from the investigated cohort (EPN_PLAGL1) for whom follow-up data were available compared to TTP of 80 patients with ZFTA:RELA-fused ependymoma (EPN_ZFTA:RELA; d).

a

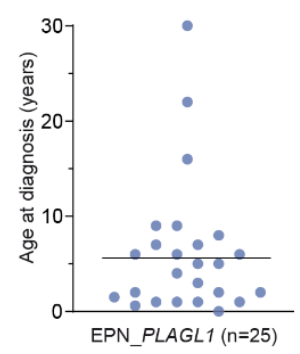

b

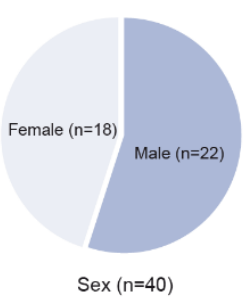

C

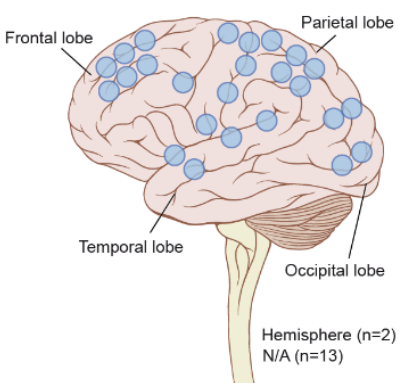

d

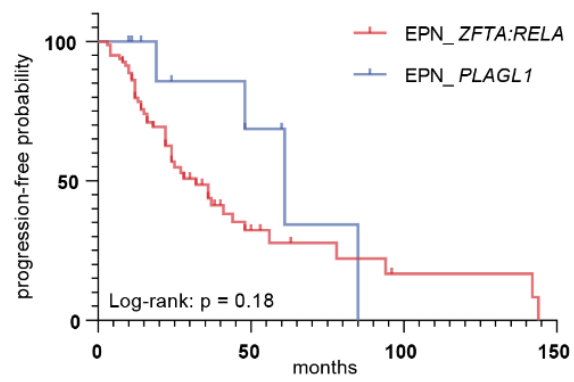


bioRxiv preprint doi: https://doi.org/10.1101/2021.04.23.441059; this version posted April 23, 2021. The copyright holder for this preprint (which was not certified by peer review) is the author/funder. All rights reserved. No reuse allowed without permission.

Fig. 5 Morphological and immunohistochemical features of tumors within the cohort. Histologically, tumors shared a moderate to high increase in cellular density with mostly monomorphic, round to oval nuclei and often prominent microcystic changes (a-d). Perivascular pseudorosettes were observed in several of the cases, although very subtle in some the samples (a-d). Occasionally, tumor cells showed oligodendroglial morphology with perinuclear halos due to cytoplasmatic clearing (e). Immunohistochemically, tumors were GFAP-positive (f) and OLIG2- and SOX10negative (g,h). In 3/16 of the cases, a dot-like positivity for EMA was detected (i). Scale bars $200 \mu \mathrm{m}$.
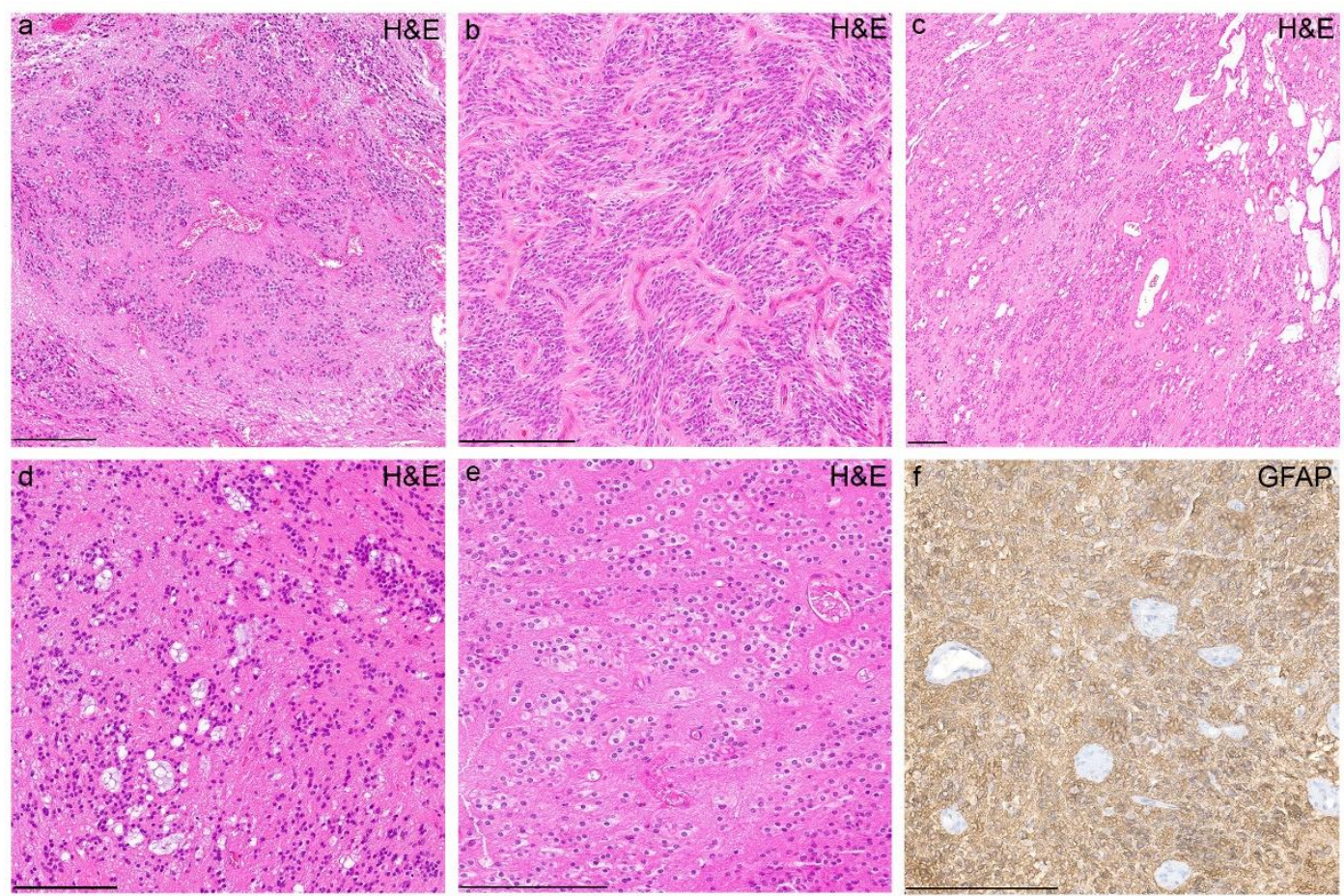

g

Olig2 $h$

SOX10

EMA

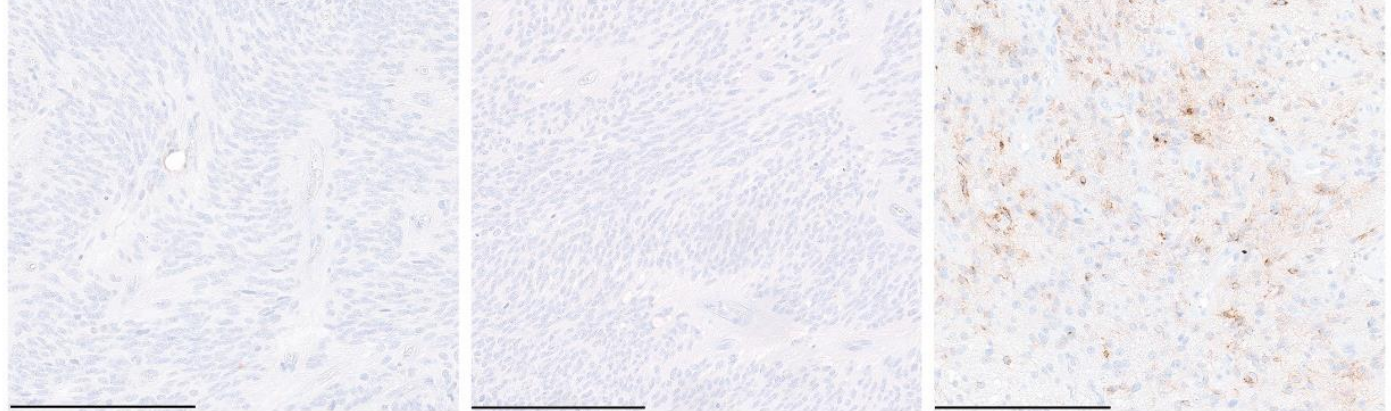

